\title{
Intercomparison of diffusion coefficient derived from the through- diffusion experiment using different numerical methods
}

\author{
Chih-Lung Chen • Tsing-Hai Wang • \\ Ching-Hor Lee $\cdot$ Shi-Ping Teng
}

Received: 6 November 2013/Published online: 2 February 2014

(c) The Author(s) 2014. This article is published with open access at Springerlink.com

\begin{abstract}
Diffusion is a dominant mechanism regulating the transport of released nuclides. The through-diffusion method is typically applied to determine the diffusion coefficients (D). Depending on the design of the experiment, the concentrations in the source term [i.e., inlet reservoir (IR)] or the end term [i.e., outlet reservoir (OR)] can be fixed or vary. The combinations involve four distinct models (i.e., the $\mathrm{CC}-\mathrm{CC}$ model, $\mathrm{CC}-\mathrm{VC}$ model, $\mathrm{VC}-$ $\mathrm{CC}$ model, and the VC-VC model). Studies discussing the VC-CC model are scant. An analytical method considering the decay effect is required to accurately interpret the radioactive nuclide diffusion experiment results. Therefore, we developed a $\mathrm{CC}-\mathrm{CC}$ model and a $\mathrm{CC}-\mathrm{VC}$ model with a decay effect and the simplified formulas of these two models to determine the diffusion coefficient (i.e., the $\mathrm{CC}-$ $\mathrm{CC}$ method and $\mathrm{CC}-\mathrm{VC}$ method). We also proposed two simplified methods using the $\mathrm{VC}-\mathrm{VC}$ model to determine the diffusion coefficient straightforwardly based upon the concentration variation in IR and OR. More importantly, the best advantage of proposed method over others is that one can derive three diffusion coefficients based on one run of experiment. In addition, applying our $\mathrm{CC}-\mathrm{VC}$ method to those data reported from Radiochemica Acta 96:111-117,
\end{abstract}

C.-L. Chen $\cdot$ T.-H. Wang $\cdot$ S.-P. Teng

Department of Engineering and System Science, National Tsing

Hua University, Hsinchu, Taiwan

\section{C.-L. Chen ( $\square)$}

Division of Chemical Engineering, Institute of Nuclear Energy

Research, Taoyuan, Taiwan

e-mail: chencl@iner.gov.tw

\section{C.-H. Lee}

Department of Medical Imaging and Radiological Sciences, I-Shou University, Kaohsiung, Taiwan
2008; and J Contam Hydrol 35:55-65, 1998, derived comparable diffusion coefficient lying in the identical order of magnitude. Furthermore, we proposed a formula to determine the conceptual critical time $(T \mathrm{c})$, which is particularly beneficial for the selection of using $\mathrm{CC}-\mathrm{VC}$ or VC-VC method. Based on our proposed method, it becomes possible to calculate diffusion coefficient from a through-diffusion experiment in a shorter period of time.

Keywords Decay effect · Through-diffusion - Diffusion coefficient $\cdot$ Radionuclide

\section{Introduction}

Diffusion is a dominant mechanism regulating the transport of released nuclides from the near field of the final disposal repository site. Diffusion constants are typically obtained using diffusion experiments. Among the numerous techniques currently available, the through-diffusion method is popularly applied for determining the diffusion coefficients $[1,2]$. The through-diffusion method is applied when the geological medium (i.e., specimen) is surrounded by two reservoirs, where one reservoir contains a concentration of nuclide (i.e., the source term) and the other reservoir is nuclide-free (i.e., the end term). The source term is known as an injective reservoir or inlet reservoir (IR), and the end term is known as a diffusive reservoir (DR) or outlet reservoir (OR).

Depending on the design of the experiment, the concentration in the source term can be fixed (i.e., a constant concentration source) or vary (i.e., a variable concentration source). Similarly, the concentration in the end term can be fixed or variable. The combinations involve a constant inlet concentration-constant outlet concentration model (CC- 
CC model) [3, 4], a constant inlet concentration-variable outlet concentration model (CC-VC model) $[5,6]$, a variable inlet concentration-constant outlet concentration model (VC-CC model) [3], and a variable inlet concentration-variable outlet concentration model (VC-VC model) [7-9]. The estimated methods of the diffusion coefficients corresponding to the models are termed the CC-CC method, $\mathrm{CC}-\mathrm{VC}$ method, $\mathrm{VC}-\mathrm{CC}$ method, and $\mathrm{VC}-\mathrm{VC}$ method, respectively. Among these models, the VC-CC model is rarely designed and performed. Certain studies have presented overviews and discussed the differences of those models, and the diffusion coefficient estimated methods were also provided $[2,10,11]$. However, these studies have not considered the decay effect in the radioactive nuclide diffusion experiment. The diffusion experiment is typically time-consuming, and the decay effect should be considered. Each experiment is necessary for a reasonable parameter estimation method [10]. In this study, we developed a CC-CC model and a CC-VC model with decay effect, as well as a parameter estimation method (CC-CC method and CC-VC method) for helping calculate diffusion coefficient by experimental researchers.

Experimental researchers specialize in experimental operations, and may overlook the fact that parameter estimation should be coordinated with the design of the experiment. For example, the design of the experiment may require a $\mathrm{CC}-\mathrm{VC}$ model, but the $\mathrm{CC}-\mathrm{CC}$ method is applied for parameter estimation. A significant difference exists in the intrinsic diffusion coefficient derived using varied through-diffusion solution methods. For this issue, we discussed the differences from various methods for distinct models by using numerical experiments [12].

In the $\mathrm{VC}-\mathrm{VC}$ model, after laborious and time-consuming one only gains a diffusion coefficient value from measuring the nuclide concentration difference between IR and OR. This is currently a resources waste. The literatures [7-9] did not apply those useful data. Thus, we develop the diffusion coefficient estimated methods from the concentration distributions of the IR and OR in the VC-VC model. This benefits for cross comparison of the estimated diffusion coefficient and enhances experiment effectiveness.

\section{Methodology}

A 1D diffusion equation derived from mass balance is adopted to describe the solute diffusion transport in the porous medium by using the following equation

$D_{p} \frac{\partial^{2} C}{\partial x^{2}}-\frac{\rho_{b}}{n} \frac{\partial S}{\partial t}=\frac{\partial C}{\partial t}$

where $C$ is the solute concentration in the pore water $\left[\mathrm{M} / \mathrm{L}^{3}\right]$; $D_{\mathrm{p}}$ is theIntrinsic diffusion coefficient in the pore water
$\left[\mathrm{L}^{2} / \mathrm{T}\right]$; $S$ is the mass of solute absorbed per unit bulk dry mass of the porous medium $[-] ; n$ is the porosity of the porous medium $[-] ; \rho_{\mathrm{b}}$ is the bulk dry density of the porous medium $\left[\mathrm{M} / \mathrm{L}^{3}\right] ; x$ is the length coordinate $[\mathrm{L}] ; t$ is the time $[\mathrm{T}]$

The first term on the left-hand side of (1) describes diffusion in the mobile pore water. The second term describes the solute absorbed by the medium. The term on the right-hand side of (1) describes the accumulation of the solute.

Assuming that sorption follows a linear relationship of $S=K_{\mathrm{d}} C$, where $K_{\mathrm{d}}$ is the distribution coefficient $\left[\mathrm{L}^{3} / \mathrm{M}\right]$, (1) can be reduced to

$D_{p} \frac{\partial^{2} C}{\partial x^{2}}=\frac{\partial C}{\partial t}\left(1+\frac{\rho_{b}}{n} K_{d}\right)$

or

$D \frac{\partial^{2} C}{\partial x^{2}}=\frac{\partial C}{\partial t}$

where $D$ is the apparent diffusion coefficient $\left[\mathrm{L}^{2} / \mathrm{T}\right][D$ can be expressed as $D=\frac{D_{p}}{R}$, where $R=1+\frac{\rho_{b}}{n} K_{d}$ is the retardation factor $(-)$ ].

Considering that the dissolved solute is a radioactive nuclide, (3) adds a decay term and becomes

$D \frac{\partial^{2} C}{\partial x^{2}}-\lambda C=\frac{\partial C}{\partial t}$

where $\lambda$ is the decay constant $[1 / T]$ (which can be expressed as $\lambda=\ln (2) / H_{\mathrm{f}} ; H_{\mathrm{f}}$ is the half-life [T]).

Equation (4) is the governing equation of the 1D diffusion model with the decay effect.

The initial equation is

$C(x, t=0)=0$

Various diffusion experiment types have different assumptions of boundary conditions and estimated methods for diffusion coefficients.

CC-CC model

\section{Experimental concept}

The boundaries of the CC-CC model with the consideration of decay effect are

$$
\left\{\begin{array}{l}
C(x=0, t)=C_{o} \mathrm{e}^{-\lambda t} \\
C(x=L, t)=0
\end{array}\right.
$$

where $C_{\mathrm{o}}$ is the initial concentration in the $\mathrm{IR}\left[\mathrm{M} / \mathrm{L}^{3}\right]$, and $L$ is the thickness of the specimen $[L]$.

The experimental concept of the CC-CC model is demonstrated in Fig. 1a. The solute concentration in the IR (lefthand side) is assumed constant while the solute concentration in the OR (right-hand side) should always be zero all the 
(a)

\begin{tabular}{|c|c|c|}
\hline Inlet Reservior(IR) & \\
$\mathrm{C}(0, \mathrm{t})=\mathrm{C}_{0} \mathrm{e}^{-\lambda \mathrm{t}}$ & $\mathrm{A}_{\mathrm{e}}$ & Outlet Reservior(OR) \\
& & $\mathrm{C}(\mathrm{L}, \mathrm{t})=0$ \\
& & $\mathrm{~V}_{\mathrm{B}}$ \\
\hline
\end{tabular}

(b)

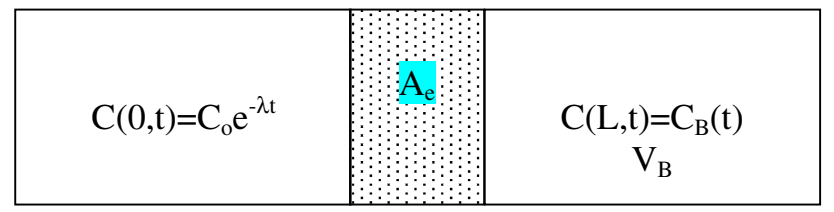

(c)

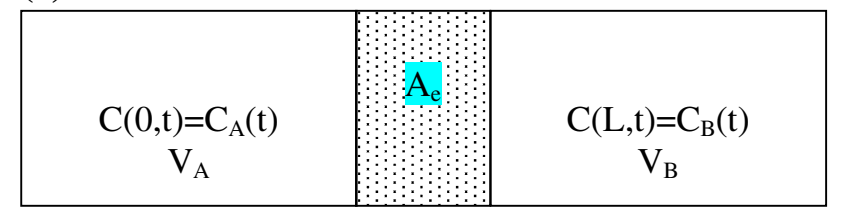

Fig. 1 Schematic diagram of three various through-diffusion models. a CC-CC Model, b CC-VC Model, c VC-VC Model

time. However, when conducting a diffusion experiment, the solute concentration in the IR would actually decrease because the radioactive nuclides will decay and diffuse into the media. The solute concentration in the right-hand side (OR) would change as well because certain amount of solute have diffused through the media. In order to keep the experimental conditions meet the boundary condition of CC-CC model, one must periodically add additional solute into IR and periodically replace the solution in the OR. From this point of view, a practical alternative is to greatly increase the volume of both IR and OR so that the varying solute concentration may be reasonably assumed negligible.

\section{Analytical solution}

The solute migration equation of the $\mathrm{CC}-\mathrm{CC}$ model in the $\mathrm{OR}$ is [3]

$C=C_{o}\left(1-\frac{x}{L}-\sum_{n=1}^{\infty} \frac{2}{n \pi} \sin \frac{n \pi x}{L} \mathrm{e}^{-\frac{D^{2} \pi^{2}}{L^{2}} t}\right)$

where $n=1,2, \ldots, \infty$.

With the decay effect, the equation can be expressed as a multiple of $\mathrm{e}^{-\lambda t}$; that is,

$C=C_{o}\left(1-\frac{x}{L}-\sum_{n=1}^{\infty} \frac{2}{n \pi} \sin \frac{n \pi x}{L} \mathrm{e}^{-\frac{D n^{2} \pi^{2}}{L^{2}} t}\right) \mathrm{e}^{-\lambda t}$

Equation (8) satisfies the governing Eq. (4), initial condition (5), and boundary conditions (6). This proves that
(8) is the analytical solution of the CC-CC model with a decay effect.

\section{CC-CC method for estimating $D$}

The procedures for estimating the apparent diffusion coefficient of the CC-CC model with a decay effect are as follows. The flux at $x=L$ can be expressed by

$$
J(L, t)=-\left.D \frac{\partial C}{\partial x}\right|_{x=L}=C_{o} \frac{D}{L}\left[1+2 \sum_{n=1}^{\infty}(-1)^{n} \mathrm{e}^{-\frac{D n^{2} \pi^{2}}{L^{2}} t}\right] \mathrm{e}^{-\lambda t}
$$

The total quantity, $\mathrm{Q}(\mathrm{t})$, diffused through the media with an effective cross-area $\left(A_{\mathrm{e}}\right)$ of up to time $\mathrm{t}$ can be calculated as

$$
\begin{aligned}
Q(t)= & \int_{0}^{t} J(L, t) A_{\mathrm{e}} d t=\frac{D A_{\mathrm{e}} C_{o}}{L}\left\{-\frac{1}{\lambda} \mathrm{e}^{-\lambda t}\right. \\
& +\frac{L}{2 \sqrt{\lambda D}}\left[\operatorname{coth}\left(\frac{L}{2} \sqrt{\frac{\lambda}{D}}\right)-\tanh \left(\frac{L}{2} \sqrt{\frac{\lambda}{D}}\right)\right] \\
& \left.-2 \sum_{n=1}^{\infty} \frac{(-1)^{n}}{\frac{D n^{2} \pi^{2}}{L^{2}}+\lambda} \mathrm{e}^{-\left(\frac{D n^{2} \pi^{2}}{L^{2}}+\lambda\right) t}\right\}
\end{aligned}
$$

From the point of view of diffusion experiments, the concentration of solute that has diffused through the media in the OR should be determined during each solution replacement. While the solute concentration ratio into the OR can be thus experimentally determined, the numerical concentration ratio in the OR can be expressed as

$$
\begin{aligned}
\frac{C(t)}{C_{\mathrm{o}}}=\frac{Q(t)}{V_{\mathrm{B}}}= & \frac{D A_{\mathrm{e}}}{V_{\mathrm{B}} L}\left\{-\frac{1}{\lambda} \mathrm{e}^{-\lambda t}+\frac{L}{2 \sqrt{\lambda D}}\left[\operatorname{coth}\left(\frac{L}{2} \sqrt{\frac{\lambda}{D}}\right)\right.\right. \\
& \left.\left.-\tanh \left(\frac{L}{2} \sqrt{\frac{\lambda}{D}}\right)\right]-2 \sum_{n=1}^{\infty} \frac{(-1)^{n}}{\frac{D n^{2} \pi^{2}}{L^{2}}+\lambda} \mathrm{e}^{-\left(\frac{D n^{2} \pi^{2}}{L^{2}}+\lambda\right) t}\right\}
\end{aligned}
$$

where $V_{\mathrm{B}}$ is the OR volume $\left[\mathrm{L}^{3}\right]$.

As $t$ increases, the third term in (11) decreases rapidly to yield the asymptotic solution $C^{\prime}(t)$

$$
\begin{aligned}
\frac{C^{\prime}(t)}{C_{\mathrm{o}}}= & \frac{D A_{\mathrm{e}}}{V_{\mathrm{B}} L}\left\{-\frac{1}{\lambda} \mathrm{e}^{-\lambda t}+\frac{L}{2 \sqrt{\lambda D}}\left[\operatorname{coth}\left(\frac{L}{2} \sqrt{\frac{\lambda}{D}}\right)\right.\right. \\
& \left.\left.-\tanh \left(\frac{L}{2} \sqrt{\frac{\lambda}{D}}\right)\right]\right\}
\end{aligned}
$$

Equation (12) is a straight line of slope $s=-\frac{D A_{\mathrm{e}}}{\lambda V_{\mathrm{B}} L}$ with $\mathrm{e}^{-\lambda t}$. Therefore, the values of the apparent diffusion coefficient can be determined as

$D=-\frac{s \lambda V_{\mathrm{B}} L}{A_{\mathrm{e}}}$ 
CC-VC model

\section{Experimental concept}

The CC-VC model is a popular diffusion experiment model. The initial conditions are (5), and the equations are as follows:

$t=0,\left\{\begin{array}{l}C(x=0, t)=C_{o} \\ C(x=L, t)=C_{\mathrm{B}}(t)=0\end{array}\right.$

where $C_{B}$ represents the concentration in the OR $\left[\mathrm{M} / \mathrm{L}^{3}\right]$.

The boundary conditions with a decay effect are expressed as

$x=0, C(x=0, t)=C_{o} \mathrm{e}^{-\lambda t}$

$x=L,-\lambda V_{\mathrm{B}} C_{\mathrm{B}}-\left.A_{e} D \frac{\partial C}{\partial x}\right|_{x=L}=V_{\mathrm{B}} \frac{\mathrm{d} C_{\mathrm{B}}}{\mathrm{d} t}$

Figure $1 \mathrm{~b}$ shows the experimental concept of the $\mathrm{CC}-$ $\mathrm{VC}$ model. The constant concentration in the IR decreases with the decay effect. In a practical experiment, one increases the volume or the initial concentration in the IR similar to the CC-CC model for increasing the solute in the IR. In the OR, the concentration increases with time, which is different from the $\mathrm{CC}-\mathrm{CC}$ model (in which the $\mathrm{OR}$ is maintained at a zero concentration). The concentration in the $\mathrm{OR}$ is measured periodically during the experiment.

\section{Solution}

\section{(1) Semi-analytical solution}

The Laplace transform method was used to develop the concentration formula with a decay effect for the specimen and the OR. The concentrations in the Laplace domain are

$\bar{C}(x, p)=\frac{C_{\mathrm{o}}}{p+\lambda}\left[\cosh (m x)-\frac{M \sinh (m L)+\cosh (m L)}{M \cosh (m L)+\sinh (m L)} \sinh (m x)\right]$

$\overline{C_{\mathrm{B}}}(p)=\frac{C_{\mathrm{o}}}{p+\lambda} \frac{M}{M \cosh (m L)+\sinh (m L)}$

where $M=\frac{m A_{\mathrm{e}} D}{V_{\mathrm{B}}(p+\lambda)}$ and $m=\sqrt{\frac{p+\lambda}{D}}$.

Equations (17) and (18) respectively satisfy the governing Eq. (4), initial condition (5), and boundary conditions (15) and (16) in the Laplace domain. These prove that (17) is a semi-analytical solution of the $\mathrm{CC}-\mathrm{VC}$ model, and that (18) is the concentration formula in the OR.

(2) Analytical solution

Similar to the discussion by Chen et al. [9], the multicompartment (MC) model was used to develop the analytical solution of the CC-VC model with a decay effect. The derivation steps are presented below.

Starting with the consideration of Fick laws, $\left(J=-D \frac{\partial C}{\partial x}\right)$, together with the assumption that the specimen was adjacent to one compartment, the mass balance of the nuclide between each compartment is derived and rearranged as follows:

$\left\{\begin{array}{l}V_{1} \frac{\mathrm{d} C}{\mathrm{~d}_{t}}=2 \alpha C_{\mathrm{B}}-\left(4 \alpha+\lambda V_{1}\right) C+2 \alpha C_{\mathrm{o}} e^{-\lambda t} \\ V_{\mathrm{B}} \frac{\mathrm{d} C_{\mathrm{B}}}{\mathrm{d} t}=2 \alpha C-\left(2 \alpha+\lambda V_{\mathrm{B}}\right) C_{\mathrm{B}}\end{array}\right.$

where $V_{1}=A_{\mathrm{e}} L$ and $\alpha=\frac{A_{\mathrm{e}} D}{L}$. Using the Laplace transform and substituting the following initial conditions yields

$t=0, C_{\mathrm{B}}=C=0$

These equations can be solved in algebraic form as

$\left\{\begin{array}{l}\bar{C}_{\mathrm{B}}=\frac{2 \alpha}{2 \alpha+(p+\lambda) V_{\mathrm{B}}} \bar{C} \\ \bar{C}=\frac{\frac{2 \alpha}{p+\lambda}}{4 \alpha+(p+\lambda) V_{1}-\frac{(2 \alpha)^{2}}{2 \alpha+(p+\lambda) V_{\mathrm{B}}}} C_{\mathrm{o}}\end{array}\right.$

The Laplace inverse transformations of these equations were implemented using the symbolic-numerical software package Mathematica 8.0 [13]. The solutions obtained under the real-time domain are as follows:

In the specimen:

$C=C_{\mathrm{o}}\left[\mathrm{e}^{-\lambda t}+\frac{\alpha V_{1}}{2 \beta}\left(\gamma_{1}-\gamma_{2}\right)-\frac{1}{2}\left(\gamma_{1}+\gamma_{2}\right)\right]$

In the OR:

$C_{\mathrm{B}}=C_{\mathrm{o}}\left[\mathrm{e}^{-\lambda t}+\frac{\alpha}{2 \beta}\left(V_{1}+2 V_{\mathrm{B}}\right)\left(\gamma_{1}-\gamma_{2}\right)-\frac{1}{2}\left(\gamma_{1}+\gamma_{2}\right)\right]$

where

$\begin{aligned} \gamma_{1} & =\mathrm{e}^{-\left(\lambda+\frac{2 \alpha}{V_{1}}+\frac{\alpha}{V_{\mathrm{B}}}+\frac{\beta}{V_{1} V_{\mathrm{B}}}\right) t}, \gamma_{2}=\mathrm{e}^{-\left(\lambda+\frac{2 \alpha}{V_{1}}+\frac{\alpha}{V_{\mathrm{B}}}-\frac{\beta}{V_{1} V_{\mathrm{B}}}\right) t}, \text { and } \beta \\ & =\sqrt{\alpha^{2}\left(V_{1}^{2}+4 V_{\mathrm{B}}^{2}\right)} .\end{aligned}$

$C C-C V$ method for estimating $D$

Without the decay effect of the CC-VC model, an proposed asymptotic solution for estimating $\mathrm{D}$ by using the following expression [14]:

$\frac{C_{\mathrm{B}}(t)}{C_{\mathrm{o}}}=1-\mathrm{e}^{-\frac{A_{\mathrm{e}} D}{V_{\mathrm{B}} L} t}$

This formula works only when the decay effect is negligible. We incorporated the decay effect into this study as shown in the analytical solutions (22). The derivation procedures are as follows:

First, we assume that $V_{\mathrm{B}} \gg V_{1}$ and $t \gg 0$. Then, 
$\beta=\sqrt{\alpha^{2}\left(V_{1}^{2}+4 V_{\mathrm{B}}^{2}\right)} \approx 2 \alpha V_{\mathrm{B}}$

$\gamma_{1}=\mathrm{e}^{-\left(\lambda+\frac{2 \alpha}{V_{1}}+\frac{\alpha}{V_{\mathrm{B}}}+\frac{\beta}{V_{1} V_{\mathrm{B}}}\right) t} \approx 0$

$\gamma_{2}=\mathrm{e}^{-\left(\lambda+\frac{2 \alpha}{V_{1}}+\frac{\alpha}{V_{\mathrm{B}}}-\frac{\beta}{V_{1} V_{\mathrm{B}}}\right) t} \approx \mathrm{e}^{-\left(\lambda+\frac{\alpha}{V_{\mathrm{B}}}\right) t}$.

Therefore,

$\frac{C_{\mathrm{B}}(t)}{C_{o}}=\left(1-\mathrm{e}^{-\frac{A_{\mathrm{e}} D}{V_{\mathrm{B}} L}}\right) \mathrm{e}^{-\lambda t}$

After arrangement, this can be expressed as

$\ln \left[\frac{C_{o}-C_{\mathrm{B}}(t) \cdot \mathrm{e}^{\lambda t}}{C_{\mathrm{o}}}\right]=-\frac{A_{\mathrm{e}} D}{V_{\mathrm{B}} L} t$

Equation (25) shows that $\ln \left[\frac{C_{o}-C_{\mathrm{B}}(t) \cdot \mathrm{e}^{\mathrm{\lambda} t}}{C_{\mathrm{o}}}\right]$ varies with $\mathrm{t}$ with a constant slope (s). Therefore, D can be determined as

$D=-\frac{s V_{\mathrm{B}} L}{A_{\mathrm{e}}}$

VC-VC model

\section{Experimental concept}

The initial condition of the VC-VC model includes (5) and the following equations:

$t=0,\left\{\begin{array}{l}C(x=0, t)=C_{\mathrm{A}}(t)=C_{o} \\ C(x=L, t)=C_{\mathrm{B}}(t)=0\end{array}\right.$

where $C_{A}$ represents the concentration in the IR $\left[M / L^{3}\right]$.

The boundary conditions with the decay effect are expressed as

$x=0,\left\{\begin{array}{l}C(x=0, t)=C_{\mathrm{A}}(t)=C_{\mathrm{o}} \mathrm{e}^{-\lambda t} \\ -\lambda V_{\mathrm{A}} C_{\mathrm{A}}+\left.A_{\mathrm{e}} D \frac{\partial C}{\partial x}\right|_{x=0}=V_{\mathrm{A}} \frac{\mathrm{d} C_{\mathrm{A}}}{\mathrm{d}_{t}}\end{array}\right.$

$x=L,\left\{\begin{array}{l}C(x=L, t)=C_{\mathrm{B}}(t) \\ -\lambda V_{\mathrm{B}} C_{\mathrm{B}}-\left.A_{\mathrm{e}} D \frac{\partial C}{\partial x}\right|_{x=L}=V_{\mathrm{B}} \frac{\mathrm{d} C_{\mathrm{B}}}{\mathrm{d}_{t}}\end{array}\right.$

where $V_{\mathrm{A}}$ is the volume of the IR $\left[\mathrm{L}^{3}\right]$.
The experimental concept is similar to that showed in Fig. 1c. The known concentration is injected into the IR from the beginning of the experiment. The concentration in the IR declines with the decay effect and diffuses through the specimen into the OR. The concentration in the OR varies with time. During the experiment, maintaining a constant concentration or increasing the solute in the IR is unnecessary. In addition, one only periodically measures concentrations in the IR and OR during the experiment. Measuring the IR concentration is the major difference between this model and the $\mathrm{CC}-\mathrm{VC}$ model. Although the measuring process of the concentration of the IR may spend resources, one can successfully estimate and confirm the diffusion coefficient, which is discussed in the following section.

\section{Solution}

\section{(1) Semi-analytical solution}

The VC-VC model with a decay effect can refer to the Ref. [9]. The semi-analytical solutions using the advectiondispersion (AD) model in the Laplace domain are as follows:

$$
\begin{aligned}
\bar{C}_{\mathrm{A}}(p)= & \frac{M_{2} V_{\mathrm{A}} C_{\mathrm{o}}}{(p+\lambda) M_{2} V_{\mathrm{A}}-(p+\lambda) M_{1} V_{\mathrm{B}}} \\
\bar{C}_{\mathrm{B}}(p)= & \frac{V_{\mathrm{A}} C_{o}}{(p+\lambda) M_{2} V_{\mathrm{A}}-(p+\lambda) M_{1} V_{\mathrm{B}}} \\
& \times\left[M_{2} \cosh \left(m_{1} L\right)+\sinh \left(m_{1} L\right)\right]
\end{aligned}
$$

where $\quad M_{2}=-\frac{M_{1} \cosh \left(m_{1} L\right)+\sinh \left(m_{1} L\right)}{M_{1} \sinh \left(m_{1} L\right)+\cosh \left(m_{1} L\right)}, \quad M_{1}=\frac{m_{1} A_{\mathrm{e}} D}{V_{\mathrm{B}}(p+\lambda)}, \quad$ and $m_{1}=\sqrt{\frac{p+\lambda}{D}}$.

\section{(2) Analytical solution}

The analytical solutions using the multi-compartment (MC) model in the IR and OR are as follows:

In the IR:

$$
C_{\mathrm{A}}=\frac{V_{\mathrm{A}} C_{\mathrm{o}}}{V}\left\{\mathrm{e}^{-\lambda t}+\frac{\left(\gamma_{3}-\gamma_{4}\right)\left(-V_{1}^{2} V_{\mathrm{A}}+2 V_{1}^{2} V_{\mathrm{B}}-V_{1} V_{\mathrm{A}} V_{\mathrm{B}}+V_{1} V_{\mathrm{B}}^{2}-2 V_{\mathrm{A}} V_{\mathrm{B}}^{2}\right)}{2 \beta_{1} V_{\mathrm{A}}}+\frac{\beta_{1}\left(\gamma_{3}+\gamma_{4}\right)\left(V_{1}+V_{\mathrm{B}}\right)}{2 \beta_{1} V_{\mathrm{A}}}\right\}
$$

In the OR:

$$
C_{\mathrm{B}}=\frac{V_{\mathrm{A}} C_{\mathrm{o}}}{V}\left\{\mathrm{e}^{-\lambda t}+\frac{\left(\gamma_{3}-\gamma_{4}\right)\left(V_{1} V_{\mathrm{A}}+V_{1} V_{\mathrm{B}}+2 V_{\mathrm{A}} V_{\mathrm{B}}\right)-\beta_{1}\left(\gamma_{3}+\gamma_{4}\right)}{2 \beta_{1}}\right\}
$$


Table 1 Default values employed in this study

\begin{tabular}{lllllll}
\hline & $L(\mathrm{~cm})$ & $\begin{array}{l}A_{\mathrm{e}} \\
\left(\mathrm{cm}^{2}\right)\end{array}$ & $\begin{array}{l}V_{\mathrm{A}} \\
\left(\mathrm{cm}^{3}\right)\end{array}$ & $\begin{array}{l}V_{\mathrm{B}} \\
\left(\mathrm{cm}^{3}\right)\end{array}$ & $\begin{array}{l}D \\
\left(\mathrm{~cm}^{2} / \text { day }\right)\end{array}$ & $\begin{array}{l}\lambda \\
(1 / \text { day })\end{array}$ \\
\hline Case_S* & 0.2 & 5 & 100 & 100 & $1 \mathrm{E}-5$ & $\begin{array}{l}1 \mathrm{E}-20 \\
1 \mathrm{E}-5\end{array}$ \\
Case_S & & & & & & \\
Case_D+ & & & & & $1 \mathrm{E}-3$ & \\
Case_D* & & & & & $1 \mathrm{E}-4$ & \\
Case_D- & & & & & $1 \mathrm{E}-7$ & \\
\hline
\end{tabular}

where

$V=V_{\mathrm{A}}+V_{1}+V_{\mathrm{B}}$,

$\beta_{1}=\sqrt{V_{1}^{2} V_{\mathrm{A}}^{2}-2 V_{1}^{2} V_{\mathrm{A}} V_{\mathrm{B}}+V_{1}^{2} V_{\mathrm{B}}^{2}+4 V_{\mathrm{A}}^{2} V_{\mathrm{B}}^{2}}$,

$\gamma_{3}=\mathrm{e}^{-\left[\lambda+\alpha\left(\frac{1}{V_{\mathrm{A}}}+\frac{2}{V_{1}}+\frac{1}{V_{\mathrm{B}}}+\frac{\beta_{1}}{V_{\mathrm{A}} V_{1} V_{\mathrm{B}}}\right)\right] t}$,

$\gamma_{4}=\mathrm{e}^{-\left[\lambda+\alpha\left(\frac{1}{V_{\mathrm{A}}}+\frac{2}{V_{1}}+\frac{1}{V_{\mathrm{B}}}-\frac{\beta_{1}}{V_{\mathrm{A}} V_{1} V_{\mathrm{B}}}\right)\right] t}$, and $\alpha=\frac{A_{\mathrm{e}} D}{L}$.

\section{$V C-V C$ method for estimating $D$}

A simple formula for estimating the diffusion coefficient with the decay effect was presented in the Ref. [9] as follows:

$D=\frac{s-\lambda}{\frac{A_{\mathrm{e}}}{L}\left(\frac{1}{V_{\mathrm{A}}}+\frac{1}{V_{\mathrm{B}}}\right)}$

where $s$ is a constant slope of the plot of $\ln \left[\frac{C_{\mathrm{o}}}{C_{\mathrm{A}}(t)-C_{\mathrm{B}}(t)}\right]$ against $\mathrm{t}$.

In this study, we also proposed two simple formulas for estimating $\mathrm{D}$ from the concentration distribution in the IR and the OR.

Assuming that a thinner specimen is used, the thickness term of the specimen can be ignored, and the asymptotic concentration distribution in the IR and OR can be gained using a Laplace inverse transform, as follows:

In the IR:

$C_{\mathrm{A}}=\frac{V_{\mathrm{A}}}{V_{\mathrm{A}}+V_{\mathrm{B}}} C_{\mathrm{o}} \mathrm{e}^{-\lambda t}\left(1+\frac{V_{\mathrm{B}}}{V_{\mathrm{A}}} \mathrm{e}^{-\gamma t}\right)$

In the OR:

$C_{\mathrm{B}}=\frac{V_{\mathrm{A}}}{V_{\mathrm{A}}+V_{\mathrm{B}}} C_{o} \mathrm{e}^{-\lambda t}\left(1-\mathrm{e}^{-\gamma t}\right)$

where $\gamma=\frac{A_{\mathrm{e}} D}{L}\left(\frac{1}{V_{\mathrm{A}}}+\frac{1}{V_{\mathrm{B}}}\right)$.

Rearranging these equations provides the simplified formulas as the following expressions:

In the IR:

$D=-\frac{s_{\mathrm{A}} \cdot L}{A_{\mathrm{e}}} \frac{V_{\mathrm{A}} V_{\mathrm{B}}}{\left(V_{\mathrm{A}}+V_{\mathrm{B}}\right)}$

where $s_{\mathrm{A}}$ is a constant slope of the plot of $\ln \left[\left(\frac{V_{\mathrm{A}}}{V_{\mathrm{B}}}+1\right) \frac{C_{\mathrm{A}}}{C_{\mathrm{o}} \mathrm{e}^{-\lambda t}}-1\right]$ against $\mathrm{t}$.
In the OR:

$D=-\frac{s_{\mathrm{B}} \cdot L}{A_{\mathrm{e}}} \frac{V_{\mathrm{A}} V_{\mathrm{B}}}{\left(V_{\mathrm{A}}+V_{\mathrm{B}}\right)}$

where $s_{\mathrm{B}}$ is a constant slope of the plot of $\ln \left[1-\left(\frac{V_{\mathrm{B}}}{V_{\mathrm{A}}}+1\right) \frac{C_{\mathrm{B}}}{C_{\mathrm{o}} \mathrm{e}^{-\lambda t}}\right]$ against $\mathrm{t}$.

\section{Model verification}

Comparison with VC-VC model

In this section, we first validated the proposed simple formulas Eq. (37), called VC-VC IR method, and Eq. (38), called VCVC OR method, to estimate D in the VC-VC model by calculating the default values of five cases (Case_S*, Case_S, Case_D+, Case_D*, and Case_D-), as shown in Table 1 and Fig. 2, and compared them with the results of Eq. (34). After plotting the linear relationship against time (t), we acquired an approximate slope by using a linear regression. The experimental diffusion coefficients were obtained by inputting the approximate slope into Eqs. (34), 37, and 38). By comparing the obtained diffusion coefficients with the theoretical coefficients (Table 2), we could assess the validity of the proposed models.

In these cases, the value of the error from the estimated D using the proposed formulas Eqs. (37) and (38) are slightly higher than the results calculated using Eq. (34). However, they are in a reasonable error range, except Case_D-. Because of the comparatively smaller diffusion coefficient in Case_D-, significantly fewer masses diffused into the OR; the lower concentration in the OR led to higher calculation errors.

From Case_S*, which is deliberately set as an extremely small decay constant $\left(1 \mathrm{E}-20\right.$ day $\left.^{-1}\right)$ to ignore the decay effect, we demonstrated that the proposed formula can be used to calculate the diffusion coefficient without the decay effect. In this case, we also proved that in a diffusion experiment, Eq. (37) can be used as the concentration distribution in IR, and Eq. (38) can be used as the concentration distribution in OR to help calculate D.

Comparison with actual diffusion experiment

In this section, two actual through-diffusion experiments were adopted to verify our models. First experiment is the diffusion of ${ }^{125}$ I radioactive nuclide through granite core sample, which was described in the Ref. [15]. The other experiment was done in the Ref. [16]. In this experiment, the diffusion of radioactive $\mathrm{U}, \mathrm{Pu}$ and $\mathrm{Am}$ carbonate complexes through Inada granite was performed.

(1) Diffusion coefficient determination from data reported by Ref. [15] 
(a)

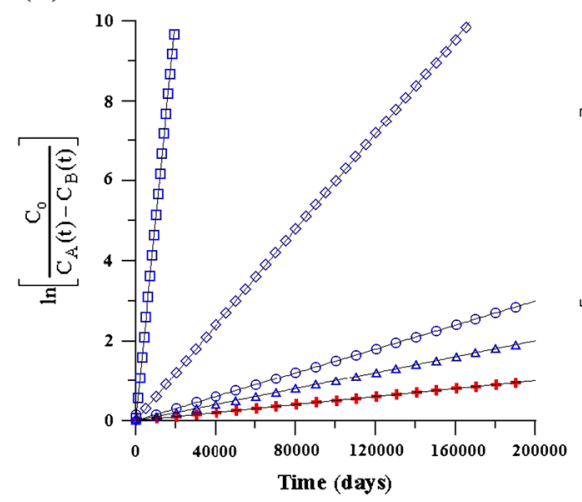

(b)

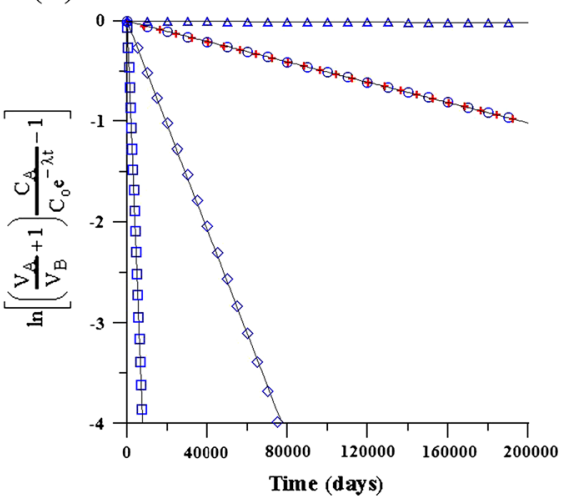

(c)

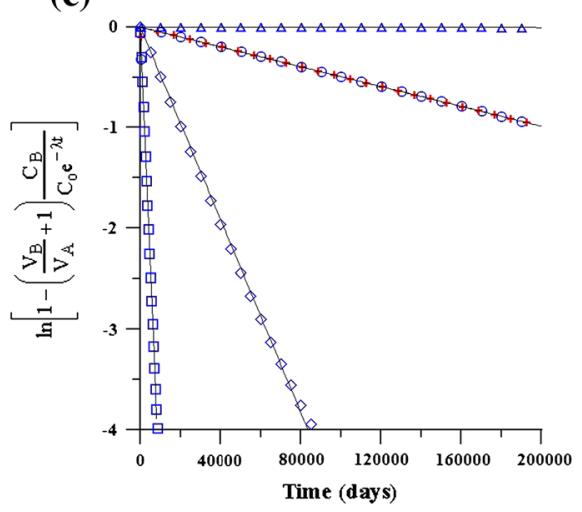

$\circ:$ Case_S $+:$ Case_S* $\square:$ Case_D $+\triangle$ :Case_D- $\diamond:$ Case_D*

Fig. 2 Estimation of D for Case_S, Case_S*, Case_D+, Case_D-, and Case_D* by Eqs. $(34,37,38)$. a VC-VC method by Eq. (34), b VC-VC IR method by Eq. (37), c VC-VC OR method by Eq. (38).
White circle:Case_S, plus sign:Case_S*, white square:Case_D+, white triangle:Case_D-, white diamond:Case_D*
The ${ }^{125}$ I nuclide with a half-life of 60.14 days is a tracer in the through-diffusion experiment conducted by Ref. [15] for determining its effective diffusion coefficient in Beishan granite. The parameter values of this experiment are listed in Table 3. Three experiments with varying sample thickness and outlet cell volume were performed. Four different analytical methods were applied to calculate effective diffusion coefficient value. The results are also shown in Table 3. According to the experimental design, the volume of injective reservoir $(\sim 1,800 \mathrm{~mL})$ is larger than the volume of DR $(\sim 60 \mathrm{~mL})$, suggesting that it is a $\mathrm{CC}-\mathrm{VC}$ model. We redrew the experimental data of Fig. 5 reported in the Ref. 15 and analyzed it by CC-VC method. After plotting $\ln \left[1-\frac{C_{\mathrm{B}}}{C_{\mathrm{o}}} \mathrm{e}^{\lambda t}\right]$ against time, a constant slope value can be obtained for calculating $\mathrm{D}$ by $\mathrm{CC}-\mathrm{VC}$ method (Eq. 26). Since the experimental data reported by $\mathrm{Lu}$ et al. [15] provide insufficient information on which cell number is used, it is assumed that the slope of fitting result suits all three experiments. The estimated results are shown in Table 3. Our results of Cell 1 and Cell 2 are consistent with those reported in the Ref. [15].

\section{(2) Diffusion coefficient determination from data reported by Ref. [16]}

The ${ }^{233} \mathrm{U},{ }^{239} \mathrm{Pu}$ and ${ }^{241} \mathrm{Am}$ radioactive nuclides were prepared as an injective source for through-diffusion experiment to determine effective diffusion coefficient in biotitic granite. The experiment was performed in triplicate using three granite disks, C15, C17 and C19. The parameter values of the experiment are summarized in Table 4 . The volumes of IR and OR in this through-diffusion experiment are the same, which makes the experimental concept similar to a VC-VC model. Since the concentration of source was kept constant during the experiment, the $\mathrm{CC}-\mathrm{VC}$ method is suitable for analyzing the concentration distribution in the OR. However, the VC-VC method was also applied to determine the diffusion coefficient for comparison.

As described by Ref. [16], no diffusion of americium through the granite was detected. The experimental data of uranium and plutonium were obtained by redrawing Fig. 2 . The diffusion coefficients of uranium and plutonium were estimated by three districted methods (i.e. the $\mathrm{CC}-\mathrm{VC}$ method, the VC-VC method and the VC-VC OR method). The analysis results of uranium are shown in Fig. 3. Figure 4 shows the analysis results of plutonium. In Figs. 3 and 4 , the concentrations distributed linearly with time for all three cells obviously. The apparent diffusion coefficients were estimated by each analytical method according to each constant slope. Then, the apparent diffusion coefficient was converted to effective diffusion coefficient by considering porosity and rock capacity factor. The calculated effective diffusion coefficients are shown in Table 4. Generally, the values analyzed in this study by CC-VC method are slightly lower, while the results estimated by VC-VC method and VC-VC OR method are somewhat larger comparing with the values reported by Ref. [16] using Crank method. Nevertheless, the obtained values by our proposed method are within experimental error.

\section{Analysis and discussion}

Model differences

Laboratory technicians are experts in the experimental method and strive to reduce the number of experimental 


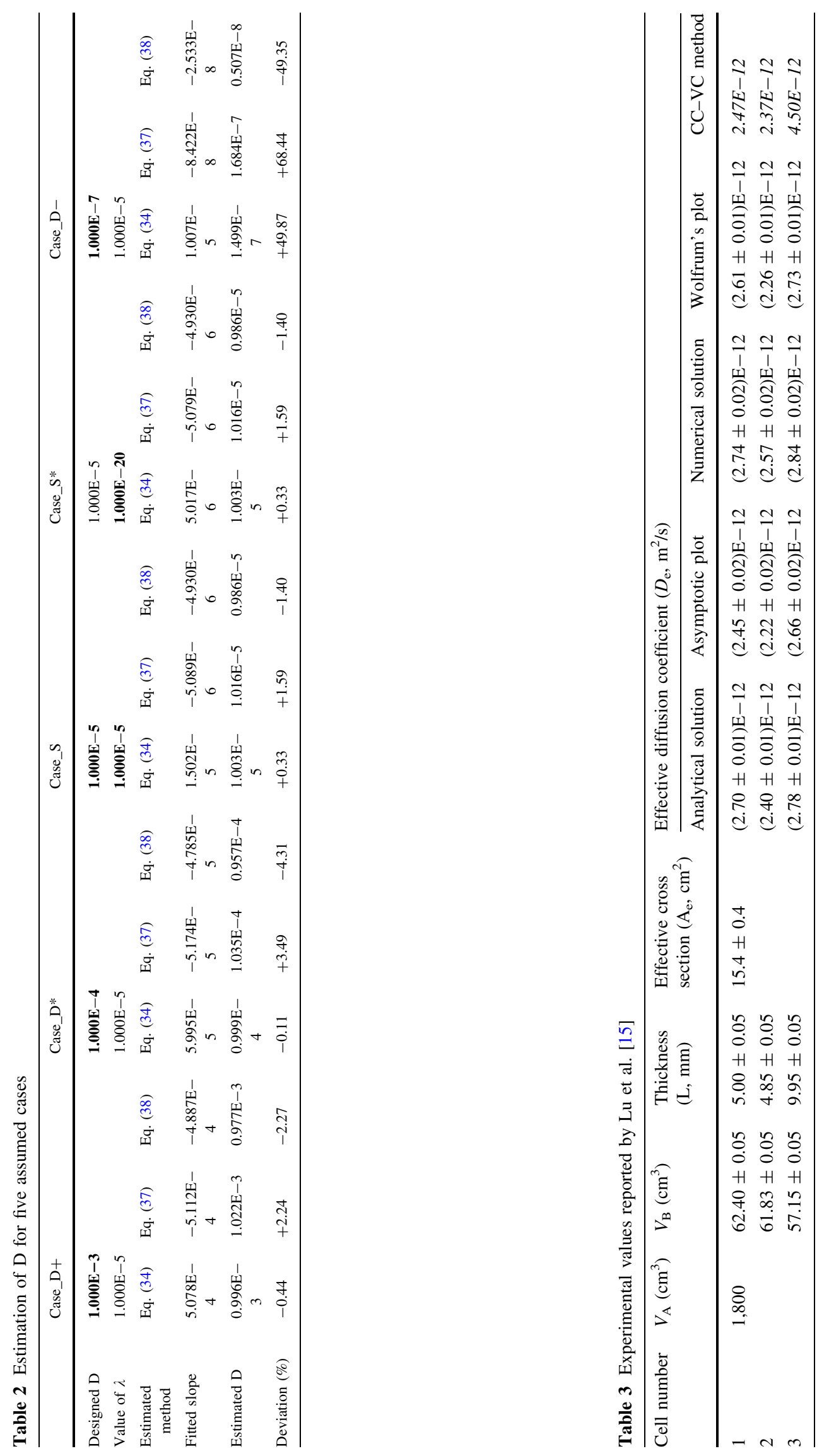




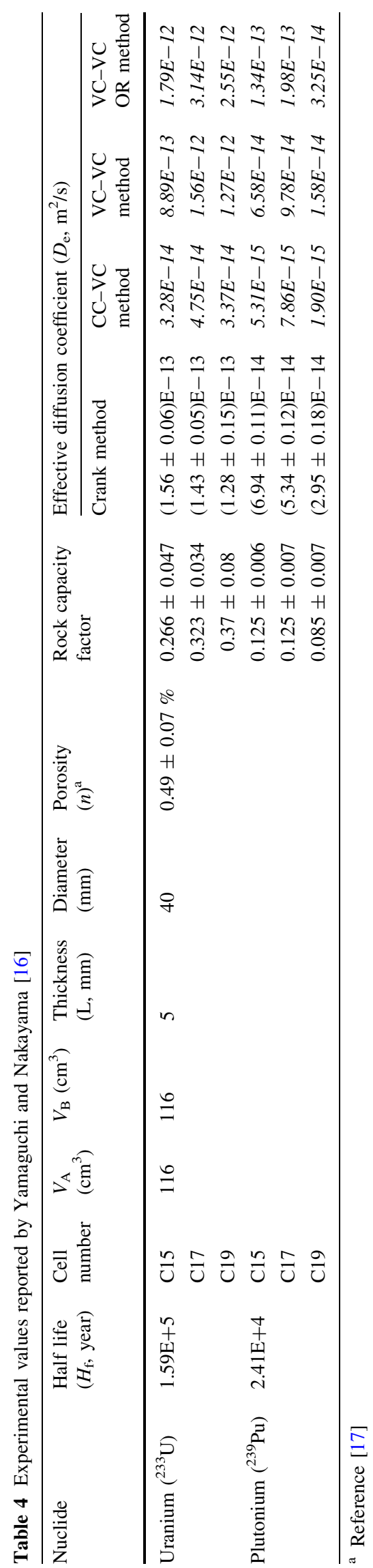

errors. However, they may make some analytical mistakes because they may adopt an inappropriate method to interpret their data. In this section, we employ Case_S and Case_D+ to validate the CC-CC method (Eq. (13)) and CC-VC method (Eq. (26)) we proposed, and also to discuss what might happen when D is estimated using an unsuitable method.

First, concentration distributions of Case_S were obtained from three numerical experiments of the $\mathrm{CC}-\mathrm{CC}$ model (Eq. (8)), CC-VC model (Eq. (18)), and the VC-VC model (Eq. (31)). The diffusion coefficient was then estimated using three distinct analysis methods (i.e., the $\mathrm{CC}-$ $\mathrm{CC}$ method, the $\mathrm{CC}-\mathrm{VC}$ method, and the VC-VC method was estimated using Eq. (34)). Figure 5 shows that the concentration distribution in the OR was comparable between numerical experiments. An approximate slope can be derived from the analysis methods and the estimated $\mathrm{D}$ can be obtained, except in the case of the $\mathrm{VC}-\mathrm{VC}$ numerical experiment, which involved estimating $\mathrm{D}$ by using the $\mathrm{CC}-\mathrm{CC}$ method or $\mathrm{CC}-\mathrm{VC}$ method, as shown in Fig. 6 and Table 5. In that case, an unreasonable result may have been obtained.

With a higher diffusion coefficient (Case_D+), the concentration distributions in the OR seem to depart from each numerical experiment (Fig. 7). After arranging for linear regression against time, the differences are obvious between each experiment (Fig. 8). A higher deviation obtained using an unsuitable analysis method is displayed clearly, as shown in Table 6.

Tables 5 and 6 show that using $\mathrm{CC}-\mathrm{CC}$ method (Eq. (13)) and CC-VC method (Eq. (26)) to determine diffusion coefficient of $\mathrm{CC}-\mathrm{CC}$ model and $\mathrm{CC}-\mathrm{VC}$ model can have a reasonable value of diffusion coefficient. The derivation is $<0.45 \%$. That proves our proposed method can calculate the diffusion coefficient with the decay effect. The analysis results also show that if an unsuitable analysis method is applied for the diffusion experiment, a similar value may be estimated. However, the parameter should be estimated using the correct analysis method for obtaining the lowest deviation.

\section{Volume ratio of IR/OR}

The CC-VC model is a special case of the VC-VC model. In the $\mathrm{VC}-\mathrm{VC}$ model, if the concentration reduction in the IR can be ignored, the VC-VC model can be considered a $\mathrm{CC}-\mathrm{VC}$ model. This condition is often based on the IR relative to the $\mathrm{OR}$, which contains large amounts of nuclides. In the experiment, the IR is designed with a larger volume, solutes are supplied circularly, or only a few nuclides diffused from the IR to the OR within the experiment period. This section clarifies which condition of the VC-VC model can be simplified to obtain the CC-VC 
(a)

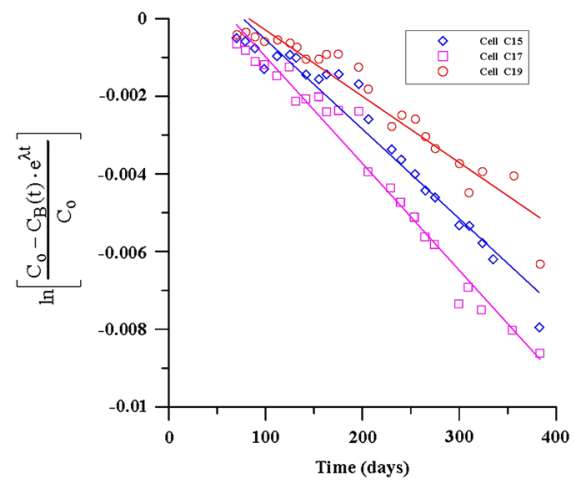

(b)

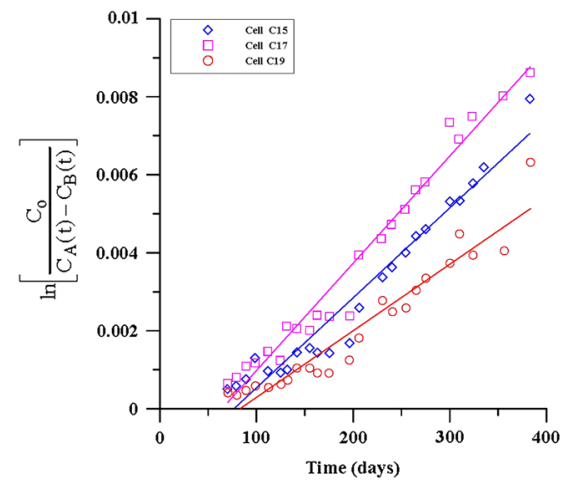

(c)

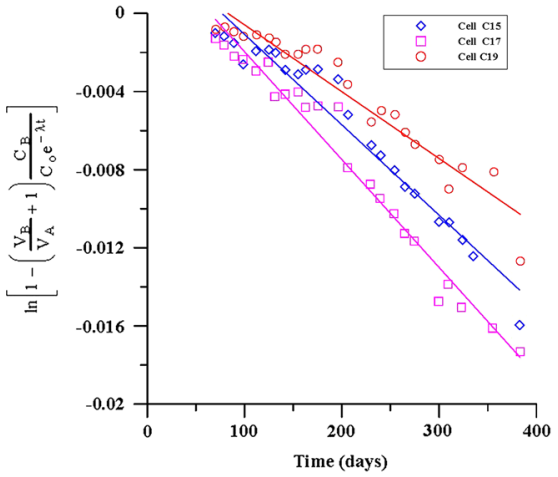

Fig. 3 Estimation of D by three distinct analysis methods with identical uranium concentration. a By CC-VC method (Eq. (26)), b by VC-VC method (Eq.(34)), c by VC-VC OR method (Eq. (38))

(a)

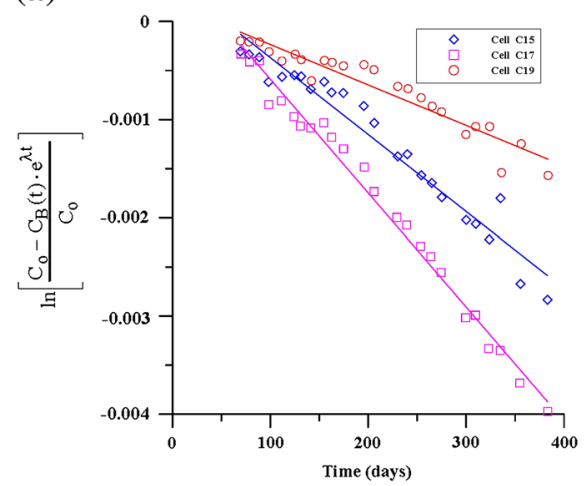

(b)

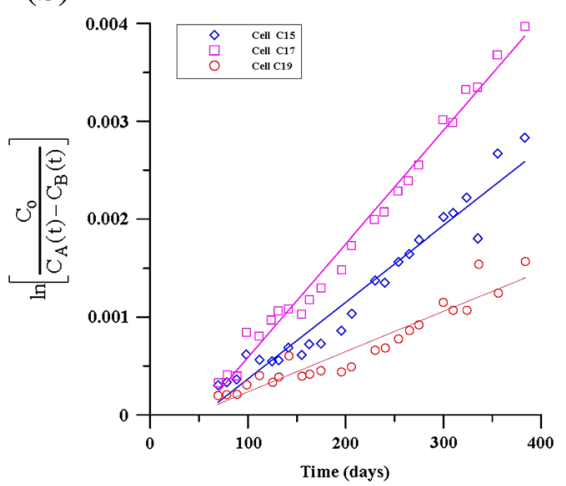

(c)

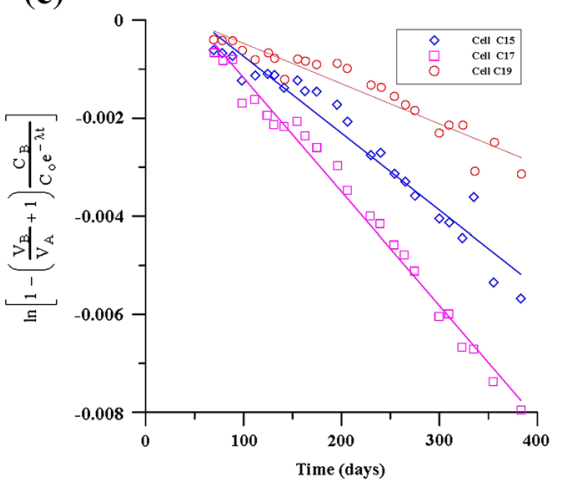

Fig. 4 Estimation of D by three distinct analysis methods with identical plutonium concentration. a By CC-VC method (Eq. (26)), b by VC-VC method (Eq. (34)), c by VC-VC OR method (Eq. (38))

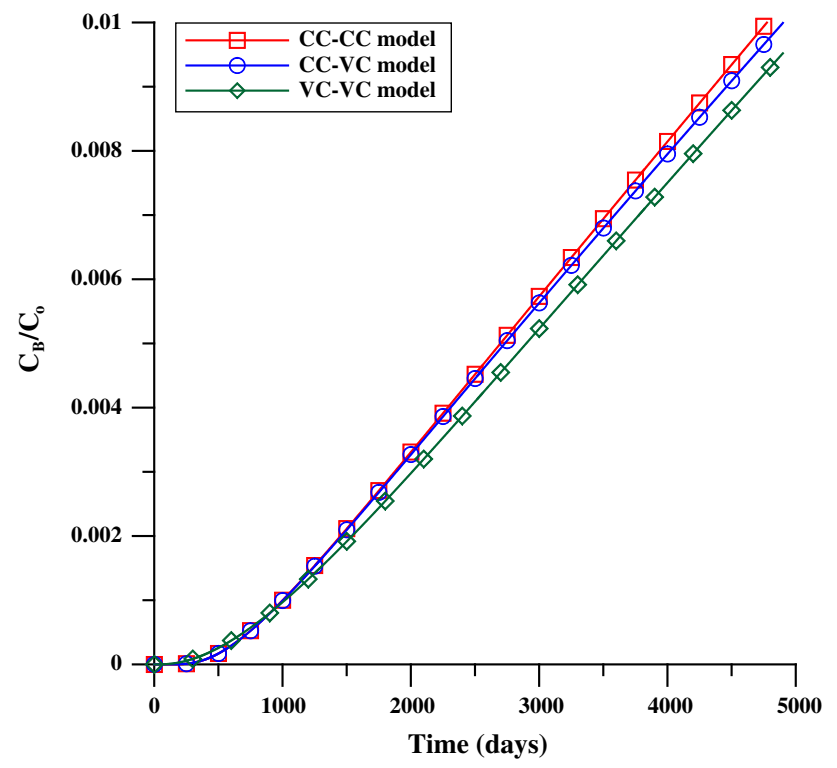

Fig. 5 The Case_S concentration distribution in the OR of CC-CC, $\mathrm{CC}-\mathrm{VC}$ and $\mathrm{VC}-\mathrm{VC}$ models model. In addition, the accuracy of the estimated D should be an evaluation criterion.

First, we used Case_S* to ignore the decay effect. Because the nuclide concentration is reduced with the decay effect in the IR or in the OR, confirming whether the concentration in the IR stays constant is difficult. The concentration distributions in the IR with a distinct volume ratio $\left(V_{\mathrm{B}} / V_{\mathrm{A}}=1 / 1,1 / 2,1 / 3,1 / 5,1 / 10\right)$ are shown in Fig. 9 . Only the declined concentration of the volume ratio of $1 / 10$ in the IR is more than $90 \%$ of the source concentration during the numerical experiment time. The concentration in the IR is reduced more slowly with a higher volume ratio. The reduced concentration in the IR of higher than $90 \%$ is assumed to be a constant concentration source; otherwise, it is a variable concentration source. Figure 9 shows that the concentration in the IR is reduced to $90 \%$ at $4.46 \mathrm{E}+4$ days of the volume ratio of 1 . The time required for the concentration to be reduced to $90 \%$ can be estimated with the following formula, which is derived from Eq. (35). 
(a)

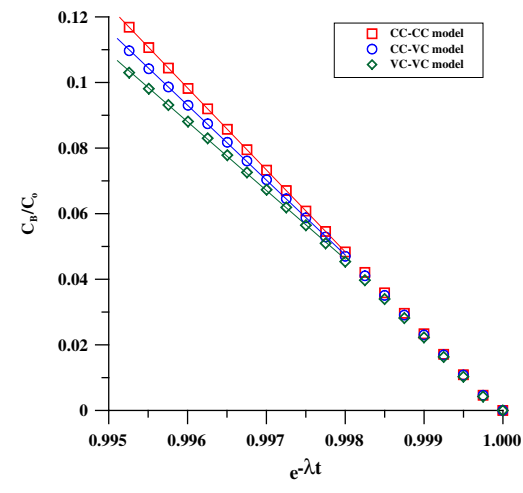

(b)

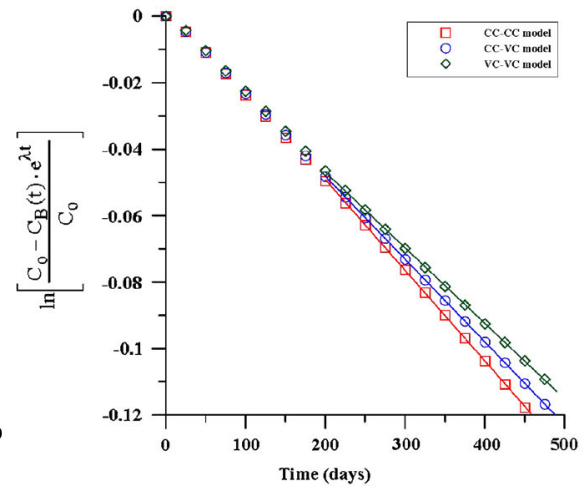

(c)

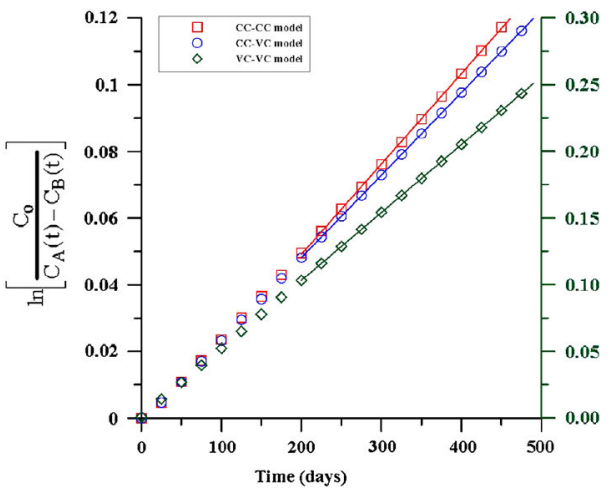

Fig. 6 Estimation of D by three distinct analysis methods of Case_S. a by CC-CC method (Eq. (13)), b by CC-VC method (Eq. (26)), $\mathbf{c}$ by VCVC method (Eq. (34))

Table 5 Estimation of D for Case_S from three distinct numerical experiments by three analysis methods

\begin{tabular}{|c|c|c|c|c|c|c|c|c|c|}
\hline \multirow{3}{*}{$\begin{array}{l}\text { Designed D } \\
\text { Numerical } \\
\text { experiment } \\
\text { Analysis } \\
\text { method }\end{array}$} & \multicolumn{9}{|l|}{$1.000 \mathrm{E}-5$} \\
\hline & \multicolumn{3}{|c|}{$\mathrm{CC}-\mathrm{CC}$ model } & \multicolumn{3}{|c|}{$\mathrm{CC}-\mathrm{VC}$ model } & \multicolumn{3}{|c|}{ VC-VC model } \\
\hline & $\begin{array}{l}\text { CC-CC } \\
\text { method }\end{array}$ & $\begin{array}{l}\mathrm{CC}-\mathrm{VC} \\
\text { method }\end{array}$ & $\begin{array}{l}\mathrm{VC}-\mathrm{VC} \\
\text { method }\end{array}$ & $\begin{array}{l}\mathrm{CC}-\mathrm{CC} \\
\text { method }\end{array}$ & $\begin{array}{l}\mathrm{CC}-\mathrm{VC} \\
\text { method }\end{array}$ & $\begin{array}{l}\text { VC-VC } \\
\text { method }\end{array}$ & $\begin{array}{l}\mathrm{CC}-\mathrm{CC} \\
\text { method }\end{array}$ & $\begin{array}{l}\mathrm{CC}-\mathrm{VC} \\
\text { method }\end{array}$ & $\begin{array}{l}\mathrm{VC}-\mathrm{VC} \\
\text { method }\end{array}$ \\
\hline Fitted slope & $-2.497 E-1$ & $-2.402 \mathrm{E}-1$ & $2.345 \mathrm{E}-1$ & $-2.586 \mathrm{E}-6$ & $-2.489 E-6$ & $-2.425 \mathrm{E}-6$ & $2.429 \mathrm{E}-6$ & $2.337 \mathrm{E}-6$ & $1.502 E-5$ \\
\hline Estimated D & $9.988 E-6$ & $9.609 \mathrm{E}-6$ & $9.382 \mathrm{E}-6$ & $1.034 \mathrm{E}-5$ & $9.955 E-6$ & $9.701 \mathrm{E}-6$ & $-3.028 E-5$ & $-3.065 \mathrm{E}-5$ & $1.003 E-5$ \\
\hline Deviation $(\%)$ & -0.12 & -3.91 & -6.18 & +3.44 & -0.45 & -2.99 & & & +0.33 \\
\hline
\end{tabular}

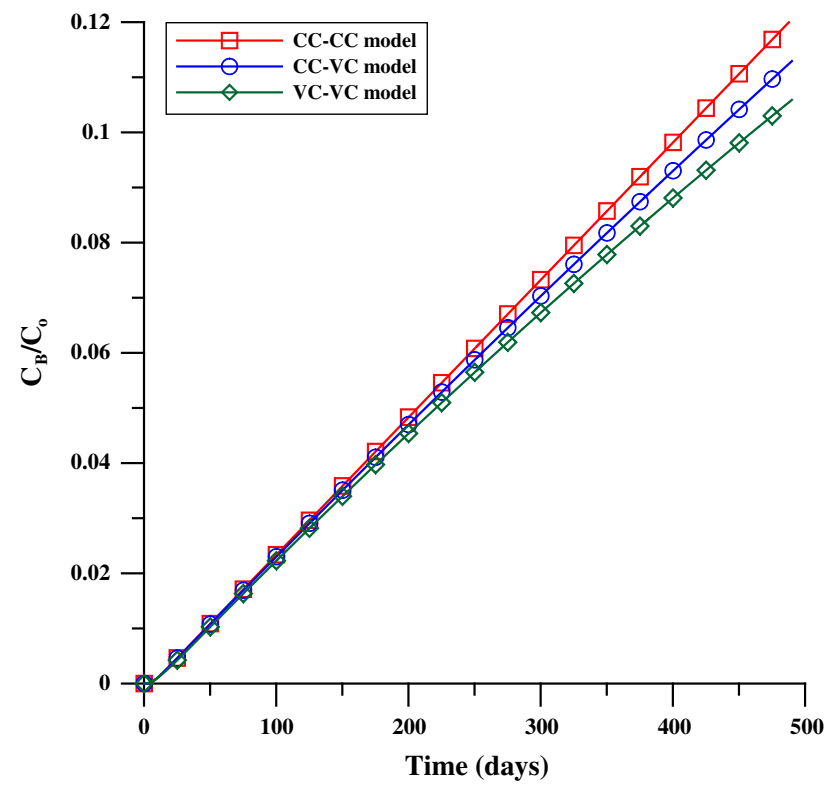

Fig. 7 The Case_D+ concentration distribution in the OR of CC$\mathrm{CC}, \mathrm{CC}-\mathrm{VC}$ and $\mathrm{VC}-\mathrm{VC}$ models

$$
T_{C}=\frac{1}{\alpha_{V_{\mathrm{B}}}\left(1+R_{V_{\mathrm{OR}} / V_{\mathrm{IR}}}\right)} \ln \left[\frac{R_{V_{\mathrm{OR}} / V_{\mathrm{IR}}}}{R_{C_{\mathrm{IR}} / C_{\mathrm{o}}}\left(1+R_{V_{\mathrm{OR}} / V_{\mathrm{IR}}}\right)-1}\right]
$$

where $\alpha_{V_{\mathrm{B}}}$ can be expressed as $\alpha_{V_{\mathrm{B}}}=\frac{\alpha}{V_{\mathrm{B}}}=\frac{A_{\mathrm{e}} D}{V_{\mathrm{B}} L}, R_{V_{\mathrm{OR}} / V_{\mathrm{IR}}}$ is the volume ratio of $\mathrm{OR}$ and IR, and $R_{C_{\mathrm{IR}} / C_{\mathrm{o}}}$ is the ratio of the declined concentration and initial concentration in the IR. In this study, $R_{C_{I R} / C_{o}}$ is assumed to be 0.9 .

The $\mathrm{T}_{\mathrm{C}} \mathrm{s}$ estimated using (39) are $9.51 \mathrm{E}+4$ days, $1.53 \mathrm{E}+5$ days, and $2.22 \mathrm{E}+5$ days, when $R_{V_{\mathrm{OR}} / V_{\mathrm{IR}}}$ is $1 / 2$, $1 / 3$, and $1 / 5$, respectively. We calculated $\mathrm{D}$ by using the $\mathrm{VC}-\mathrm{VC}$ method and the $\mathrm{CC}-\mathrm{VC}$ method with the two conditions (i.e., $t<T_{\mathrm{C}}$ or $t>T_{\mathrm{C}}$ ). The results are shown in Table 7 . The estimated D using the CC-VC model of $t<T_{\mathrm{C}}$ clearly has a deviation lower than that of $t>T_{\mathrm{C}}$, except when $R_{V_{\mathrm{OR}} / V_{\mathrm{IR}}}$ is $1 / 10$, in which case $C_{\mathrm{A}} / C_{\mathrm{o}}$ is larger than 0.9 for the duration of the numerical experiment. The deviation of the estimated $\mathrm{D}$ using the $\mathrm{CC}-\mathrm{VC}$ model of $t<T_{\mathrm{C}}$ is approximately 
(a)

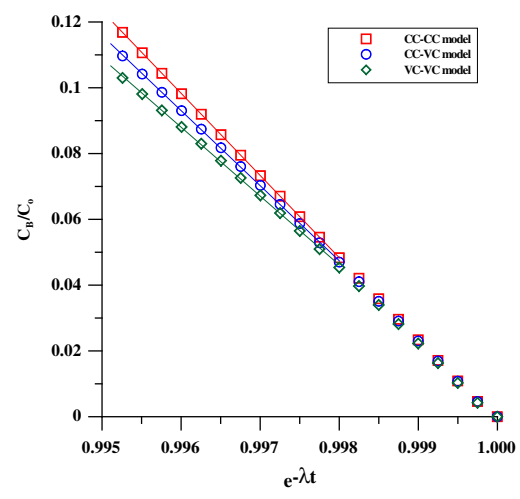

(b)

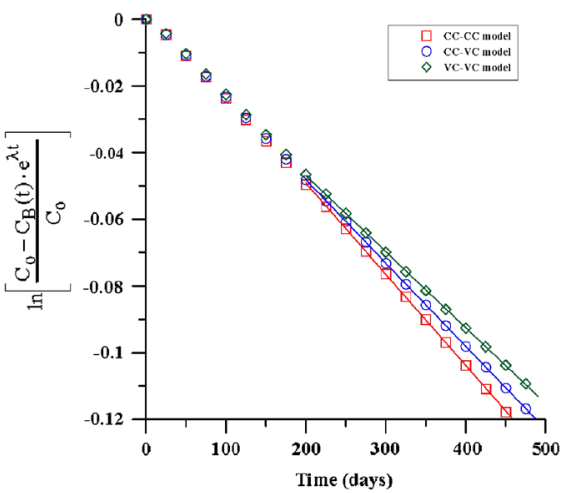

(c)

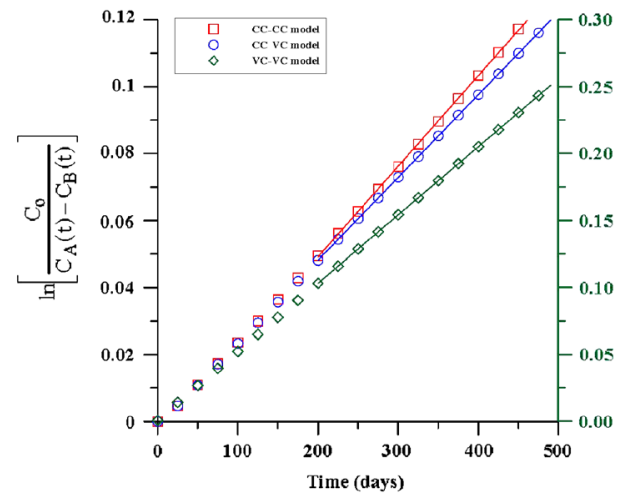

Fig. 8 Estimation of D by three distinct analysis methods of Case_D+. a By CC-CC method (Eq. (13)), b by CC-VC method (Eq. (26)), $\mathbf{c}$ by VC-VC method (Eq. (34))

Table 6 Estimation of D for Case_D+ from three distinct numerical experiments by three analysis methods

\begin{tabular}{|c|c|c|c|c|c|c|c|c|c|}
\hline \multirow{3}{*}{$\begin{array}{l}\text { Designed D } \\
\text { Numerical } \\
\text { experiment } \\
\text { Analysis } \\
\text { method }\end{array}$} & \multicolumn{9}{|l|}{$1.000 \mathrm{E}-3$} \\
\hline & \multicolumn{3}{|l|}{$\mathrm{CC}-\mathrm{CC}$} & \multicolumn{3}{|l|}{$\mathrm{CC}-\mathrm{VC}$} & \multicolumn{3}{|l|}{ VC-VC } \\
\hline & $\begin{array}{l}\mathrm{CC}-\mathrm{CC} \\
\text { method }\end{array}$ & $\begin{array}{l}\mathrm{CC}-\mathrm{VC} \\
\text { method }\end{array}$ & $\begin{array}{l}\text { VC-VC } \\
\text { method }\end{array}$ & $\begin{array}{l}\mathrm{CC}-\mathrm{CC} \\
\text { method }\end{array}$ & $\begin{array}{l}\mathrm{CC}-\mathrm{VC} \\
\text { method }\end{array}$ & $\begin{array}{l}\text { VC-VC } \\
\text { method }\end{array}$ & $\begin{array}{l}\text { CC-CC } \\
\text { method }\end{array}$ & $\begin{array}{l}\mathrm{CC}-\mathrm{VC} \\
\text { method }\end{array}$ & $\begin{array}{l}\text { VC-VC } \\
\text { method }\end{array}$ \\
\hline Fitted slope & $-2.500 E+1$ & $-2.283 \mathrm{E}+1$ & $-2.094 \mathrm{E}+1$ & $-2.729 \mathrm{E}-4$ & $-2.492 E-4$ & $-2.276 \mathrm{E}-4$ & $2.722 \mathrm{E}-4$ & $2.474 \mathrm{E}-4$ & $5.078 E-4$ \\
\hline Estimated D & $1.000 E-3$ & $9.130 \mathrm{E}-4$ & $8.377 \mathrm{E}-4$ & $1.092 \mathrm{E}-3$ & $9.968 E-4$ & $9.105 \mathrm{E}-4$ & $1.049 \mathrm{E}-3$ & $9.495 \mathrm{E}-4$ & $0.996 E-3$ \\
\hline Deviation $(\%)$ & 0.00 & -8.70 & -16.23 & +9.16 & -0.32 & -8.95 & +4.88 & -5.05 & -0.44 \\
\hline
\end{tabular}

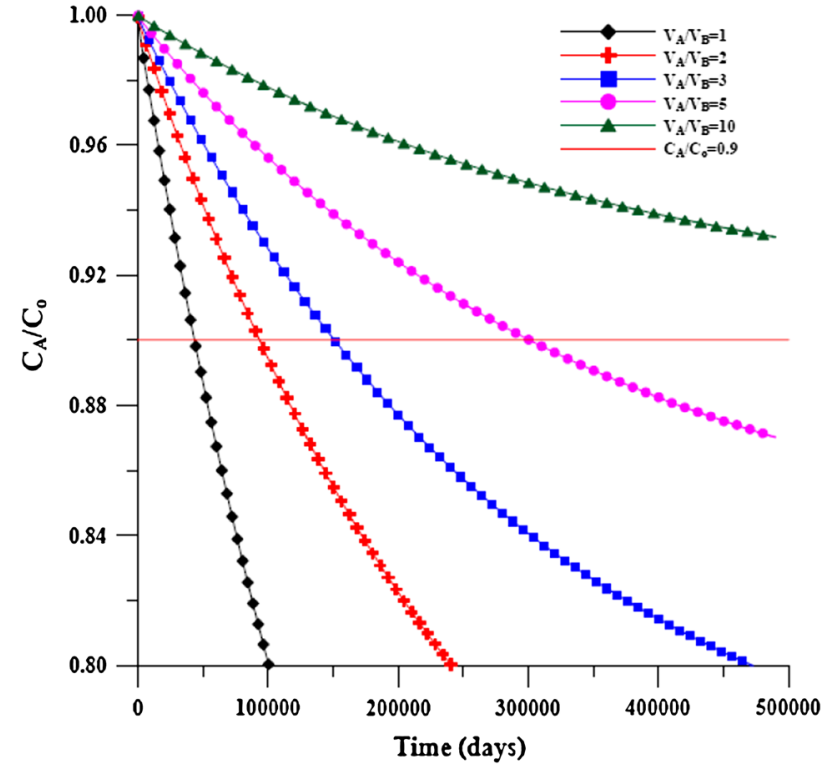

Fig. 9 Concentration distribution of Case_S* with varied volume ratio in the IR
$<7 \%$, which shows that a reasonable result can be achieved when the diffusion experiment is established as a $\mathrm{VC}-\mathrm{VC}$ model but analyzed using the $\mathrm{CC}-\mathrm{VC}$ method when the time $\mathrm{t}$ is $<T_{\mathrm{C}}$. However, the estimated $\mathrm{D}$ using the $\mathrm{VC}-\mathrm{VC}$ method has a smaller deviation than using the CC-VC method. This result shows that an experiment with a suitable analysis method should yield a more accurate parameter.

Using Case_S for the case with the decay effect, the concentration distributions in the IR with distinct $R_{V_{\mathrm{OR}} / V_{\mathrm{IR}}}$ are shown in Fig. 10. For example, when $R_{V_{\mathrm{OR}} / V_{\mathrm{IR}}}$ is $1 / 1$, the time is $<1.00 \mathrm{E}+4$, because $C_{\mathrm{A}} / C_{\mathrm{o}}=0.9$. It is difficult to distinguish whether the diffusion effect or the decay effect cause a reduction in concentration. The concentration data product $\mathrm{e}^{\lambda t}$ used to eliminate the decay effect can be used to obtain the concentration data without the decay effect, as shown in Fig. 9. The $T_{\mathrm{C}}$ can then be determined with Eq. (39), and the same result can be obtained, as noted previously. 

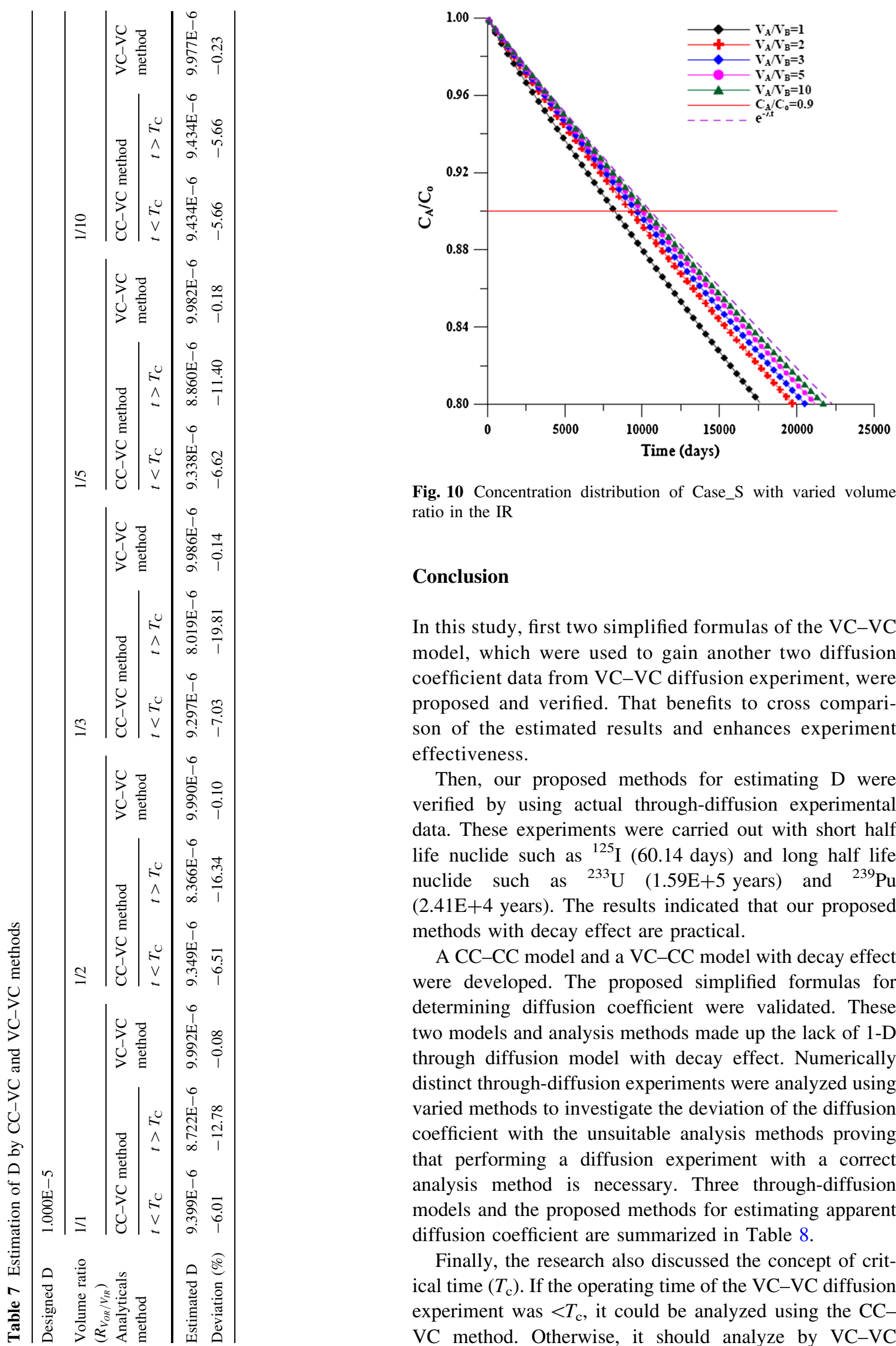

Fig. 10 Concentration distribution of Case_S with varied volume ratio in the IR

\section{Conclusion}

In this study, first two simplified formulas of the VC-VC model, which were used to gain another two diffusion coefficient data from VC-VC diffusion experiment, were proposed and verified. That benefits to cross comparison of the estimated results and enhances experiment effectiveness.

Then, our proposed methods for estimating D were verified by using actual through-diffusion experimental data. These experiments were carried out with short half life nuclide such as ${ }^{125} \mathrm{I}$ (60.14 days) and long half life nuclide such as ${ }^{233} \mathrm{U} \quad(1.59 \mathrm{E}+5$ years $)$ and ${ }^{239} \mathrm{Pu}$ $(2.41 \mathrm{E}+4$ years). The results indicated that our proposed methods with decay effect are practical.

A CC-CC model and a VC-CC model with decay effect were developed. The proposed simplified formulas for determining diffusion coefficient were validated. These two models and analysis methods made up the lack of 1-D through diffusion model with decay effect. Numerically distinct through-diffusion experiments were analyzed using varied methods to investigate the deviation of the diffusion coefficient with the unsuitable analysis methods proving that performing a diffusion experiment with a correct analysis method is necessary. Three through-diffusion models and the proposed methods for estimating apparent diffusion coefficient are summarized in Table 8.

Finally, the research also discussed the concept of critical time $\left(T_{\mathrm{c}}\right)$. If the operating time of the $\mathrm{VC}-\mathrm{VC}$ diffusion experiment was $<T_{\mathrm{c}}$, it could be analyzed using the $\mathrm{CC}-$ $\mathrm{VC}$ method. Otherwise, it should analyze by $\mathrm{VC}-\mathrm{VC}$ 
Table 8 Summary of three through-diffusion models and proposed methods for D

\begin{tabular}{|c|c|c|c|}
\hline $\begin{array}{l}\text { Diffusion } \\
\text { model }\end{array}$ & Equation & Plotting & $\mathrm{D}$ \\
\hline $\begin{array}{r}\mathrm{CC}-\mathrm{CC} \\
\text { model }\end{array}$ & $\begin{array}{l}\frac{C}{C_{o}}=\left(1-\frac{x}{L}-\sum_{n=1}^{\infty} \frac{2}{n \pi} \sin \frac{n \pi x}{L} \mathrm{e}^{-\frac{D n^{2} \pi^{2}}{L^{2}} t}\right) \mathrm{e}^{-\lambda t} \\
\frac{C_{B}}{C_{o}}=\frac{D A_{e}}{V_{B} L}\left\{-\frac{1}{\lambda} \mathrm{e}^{-\lambda t}+\frac{L}{2 \sqrt{\lambda D}}\left[\operatorname{coth}\left(\frac{L}{2} \sqrt{\frac{\lambda}{D}}\right)-\tanh \left(\frac{L}{2} \sqrt{\frac{\lambda}{D}}\right)\right]-2 \sum_{n=1}^{\infty} \frac{(-1)^{n}}{\frac{D n^{2} \pi^{2}}{L^{2}}+\lambda} \mathrm{e}^{-\left(\frac{D n^{2} \pi^{2}}{L^{2}}+\lambda\right) t}\right\}\end{array}$ & $\begin{array}{l}\text { CC-CC method } \\
\frac{C_{B}}{C_{o}} \text { v.s. } e^{-\lambda t}\end{array}$ & $D=-\frac{s \lambda V_{B} L}{A_{e}}$ \\
\hline $\begin{array}{r}\mathrm{CC}-\mathrm{VC} \\
\text { model }\end{array}$ & $\begin{array}{l}\text { (1) Semi-analytical solution } \\
\frac{\bar{C}}{C_{o}}=\frac{1}{p+\lambda}\left[\cosh (m x)-\frac{M \sinh (m L)+\cosh (m L)}{M \cosh (m L)+\sinh (m L)} \sinh (m x)\right], \frac{\bar{C}_{B}}{C_{o}}=\frac{1}{p+\lambda} \frac{M}{M \cosh (m L)+\sinh (m L)} \text { where } \\
M=\frac{m A_{e} D}{V_{B}(p+\lambda)} \text { and } m=\sqrt{\frac{p+\lambda}{D}} \\
\text { (2) Analytical solution } \\
\frac{C}{C_{o}}=\mathrm{e}^{-\lambda t}+\frac{\alpha V_{1}}{2 \beta}\left(\gamma_{1}-\gamma_{2}\right)-\frac{1}{2}\left(\gamma_{1}+\gamma_{2}\right), \frac{C_{B}}{C_{o}}=\mathrm{e}^{-\lambda t}+\frac{\alpha}{2 \beta}\left(V_{1}+2 V_{B}\right)\left(\gamma_{1}-\gamma_{2}\right)- \\
\frac{1}{2}\left(\gamma_{1}+\gamma_{2}\right) \text { where } \gamma_{1}=\mathrm{e}^{-\left(\lambda+\frac{2 \alpha}{V_{1}}+\frac{\alpha}{V_{B}}+\frac{\beta}{V_{1} V_{B}}\right) t}, \gamma_{2}=\mathrm{e}^{-\left(\lambda+\frac{2 \alpha}{V_{1}}+\frac{\alpha}{V_{B}}-\frac{\beta}{V_{1} V_{B}}\right) t} \text {, and } \\
\beta=\sqrt{\alpha^{2}\left(V_{1}^{2}+4 V_{B}^{2}\right)}\end{array}$ & $\begin{array}{l}\text { CC-VC method } \\
\ln \left[1-\frac{C_{B}}{C_{o}} \mathrm{e}^{\lambda t}\right] \text { v.s. } \mathrm{t}\end{array}$ & $D=-\frac{s V_{B} L}{A_{e}}$ \\
\hline $\begin{array}{l}\text { VC-V } \\
\text { C model }\end{array}$ & $\begin{array}{l}\text { (1) Semi-analytical solution } \\
\frac{\bar{C}_{A}}{C_{o}}=\frac{M_{2} V_{A}}{(p+\lambda) M_{2} V_{A}-(p+\lambda) M_{1} V_{B}}, \frac{\bar{C}_{B}}{C_{o}}=\frac{V_{A}}{(p+\lambda) M_{2} V_{A}-(p+\lambda) M_{1} V_{B}}\left[M_{2} \cosh \left(m_{1} L\right)+\sinh \left(m_{1} L\right)\right] \text { where } \\
M_{2}=-\frac{M_{1} \cosh \left(m_{1} L\right)+\sinh \left(m_{1} L\right)}{M_{1} \sinh \left(m_{1} L\right)+\cosh \left(m_{1} L\right)}, M_{1}=\frac{m_{1} A_{e} D}{V_{B}(p+\lambda)}, \text { and } m_{1}=\sqrt{\frac{p+\lambda}{D}} . \\
\text { (2) Analytical solution } \\
C_{A}=\frac{V_{A} C_{o}}{V}\left\{\mathrm{e}^{-\lambda t}+\frac{\left(\gamma_{3}-\gamma_{4}\right)\left(-V_{1}^{2} V_{A}+2 V_{1}^{2} V_{B}-V_{1} V_{A} V_{B}+V_{1} V_{B}^{2}-2 V_{A} V_{B}^{2}\right)}{2 \beta_{1} V_{A}}+\frac{\beta_{1}\left(\gamma_{3}+\gamma_{4}\right)\left(V_{1}+V_{B}\right)}{2 \beta_{1} V_{A}}\right\} \\
C_{B}=\frac{V_{A} C_{o}}{V}\left\{\mathrm{e}^{-\lambda t}+\frac{\left(\gamma_{3}-\gamma_{4}\right)\left(V_{1} V_{A}+V_{1} V_{B}+2 V_{A} V_{B}\right)-\beta_{1}\left(\gamma_{3}+\gamma_{4}\right)}{2 \beta_{1}}\right\} \text { where } V=V_{A}+V_{1}+V_{B}, \\
\alpha=\frac{A_{e} D}{L}, \beta_{1}=\sqrt{V_{1}^{2} V_{A}^{2}-2 V_{1}^{2} V_{A} V_{B}+V_{1}^{2} V_{B}^{2}+4 V_{A}^{2} V_{B}^{2}}, \gamma_{3}=\mathrm{e}^{-\left[\lambda+\alpha\left(\frac{1}{V_{A}}+\frac{2}{V_{1}}+\frac{1}{V_{B}}+\frac{\beta_{1}}{V_{A} V_{1} V_{B}}\right)\right] t}, \\
\text { and } \gamma_{4}=\mathrm{e}^{-\left[\lambda+\alpha\left(\frac{1}{V_{A}}+\frac{2}{V_{1}}+\frac{1}{V_{B}}-\frac{\beta_{1}}{V_{A} V_{1} V_{B}}\right)\right] t}\end{array}$ & $\begin{array}{l}\text { (1) VC-VC method } \\
\ln \left[\frac{C_{o}}{C_{A}(t)-C_{B}(t)}\right] \text { v.s. t } \\
\text { (2) VC-VC IR method } \\
\ln \left[\left(\frac{V_{A}}{V_{B}}+1\right) \frac{C_{A}}{C_{o} e^{-\lambda t}}-1\right] \\
\text { V.s. t } \\
\text { (3) VC-VC OR } \\
\text { method } \\
\ln \left[1-\left(\frac{V_{B}}{V_{A}}+1\right) \frac{C_{B}}{C_{o} e^{-\lambda t}}\right] \\
\text { V.s. t }\end{array}$ & $\begin{array}{l}D=\frac{s-\lambda}{\frac{A_{e}}{L}\left(\frac{1}{V_{A}}+\frac{1}{V_{B}}\right)} \\
D=-\frac{s_{A} \cdot L}{A_{e}} \frac{V_{A} V_{B}}{\left(V_{A}+V_{B}\right)}\end{array}$ \\
\hline
\end{tabular}

method. That is an important reference for laboratory technicians who want to calculate diffusion coefficient and decide the reasonable analysis method.

Open Access This article is distributed under the terms of the Creative Commons Attribution License which permits any use, distribution, and reproduction in any medium, provided the original author(s) and the source are credited.

\section{References}

1. Shackelford CD (1991) Laboratory diffusion testing for waste disposal-A review. J Contam Hydrol 7:177-217

2. García-Gutiérrez M, Cormenzana JL, Missanal T, Mingarro M, Molinero J (2006) Overview of laboratory methods employed for obtaining diffusion coefficients in FEBEX compacted bentonite. J Iberian Geol 32:37-53

3. Carslaw HS, Jaeger JC (1959) Conduction of heat in solids, 2nd edn. Oxford University, New York

4. Crank J (1975) The mathematics of diffusion, 2nd edn. Clarendon, Oxford

5. Tits J, Jakob A, Wieland E, Spieler P (2003) Diffusion of tritiated water and ${ }^{22} \mathrm{Na}^{+}$through non-degraded hardened cement pastes. J Contam Hydrol 61:45-62
6. Lü X, Ahl J (2005) Studying of salt diffusion coefficient in brick: analytical and numerical methods. J Mater Sci 40:3795-3802

7. Moridis GJ (1999) Semianalytical solutions for parameter estimation in diffusion cell experiments. Water Resour Res 35(6): 1729-1740

8. Bharat TV, Suvapullaiah PV, Allam MM (2009) Swarm intelligence-based solver for parameter estimation of laboratory through-diffusion transport of contaminants. Comput Geotech 36:984-992

9. Chen CL, Wang TH, Lee CH, Teng SP (2012) The development of a through-diffusion model with a parent-daughter decay chain. J Contam Hydrol 138(139):1-14

10. Takeda M, Nakajima H, Zhang M, Hiratsuka T (2008) Laboratory longitudinal diffusion tests: 1 . Dimensionless formulations and validity of simplified solutions. J Contam Hydrol 97:117-134

11. Takeda M, Zhang M, Nakajima H, Hiratsuka T (2008) Laboratory longitudinal diffusion tests: 2. Parameter estimation by inverse analysis. J Contam Hydrol 97:100-116

12. Benning JL, Barnes DL (2009) Comparison of modeling methods for the determination of effective porosities and diffusion coefficients in through-diffusion tests. Water Resour Res 45:W09419. doi:10.1029/2008WR007236

13. Wolfram Research Inc. (2012) Mathematica, Version 8.0, Champaign

14. Brace WF, Walsh JB, Frangos WT (1968) Permeability of granite under high pressure. J Geophys Res 73(6):2225-2236

15. Lu CJ, Liu CL, Chen T, Wang J, Wang XY, Su R, Sun JY, Yang RX, Zhang XS (2008) Determination of the effective diffusion 
coefficient for ${ }^{125} \mathrm{I}^{-}$in Beishan granite. Radiochemica Acta 96:111-117. doi:10.1524/ract.2008.1469

16. Yamaguchi T, Nakayama S (1998) Diffusivity of U, Pu and Am carbonate complexes in a granite from Inada, Ibaraki, Japan studied by through diffusion. J Contam Hydrol 35:55-65
17. Yamaguchi T, Nakayama S, Kawada C (2002) Contribution of the surface diffusion on mass transfer of $\mathrm{Ba}^{2+}$ in granite matrix. J Nucl Fuel Cycle Environ 9(1):61-66 